REVIEW

\title{
Exemestane in early breast cancer: a review
}

\author{
Michael Untch' \\ Christian Jackisch ${ }^{2}$ \\ 'Interdisciplinary Breast Centre, \\ Helios Klinikum Berlin-Buch, \\ University Charité, Berlin, Germany; \\ ${ }^{2}$ Department of Gynecology/ \\ Obstetrics, Klinikum Offenbach \\ $\mathrm{GmbH}$, Offenbach, Germany
}

\begin{abstract}
The adjuvant treatment of women with endocrine-sensitive early breast cancer has been dominated for the last 40 years by tamoxifen. However, the side-effects associated with this therapy have prompted a search for safer and biochemically more selective endocrine agents and led to the development of the third-generation aromatase inhibitors (AIs) anastrozole, letrozole and exemestane. Promising results in advanced disease have paved the way for treating early breast cancer, and AIs are increasingly replacing tamoxifen in the adjuvant setting. Several large, randomized trials with AIs have been completed or are ongoing in women with early-stage breast cancer, documenting the significant impact that these drugs are making on the risk for recurrence of breast cancer. As a result, there is increasing and widespread use of AI therapy for the treatment of early-stage endocrine-responsive breast cancer. This review summarizes the data for exemestane in the adjuvant setting, showing that a switch to exemestane after 2 to 3 years of tamoxifen therapy is associated with a statistically significant survival benefit and is regarded as being sensitive by international and national experts.
\end{abstract}

Keywords: early breast cancer, adjuvant setting, endocrine-sensitive, tamoxifen, aromatase inhibitor, exemestane, switch, IES 31, NSABP B-33, TEAM

Despite the improvement in survival observed with tamoxifen, a considerable proportion of women with endocrine-sensitive breast cancer do not appear to benefit from the orally active selective estrogen receptor modulator (SERM): more than half of the women experience breast cancer relapses and more than two thirds of deaths occur after the initial 5 years after surgery (Early Breast Cancer Trialists' Collaborative Group 1998, 2005, 2007).

Laboratory studies indicate that a reduction in the antagonist properties of tamoxifen could be caused by the up-regulation of tyrosine kinase receptors, especially HER2 and epidermal growth-factor receptors (EGFR), downstream protein kinases, or both, resulting in either primary or adaptive/acquired resistance to tamoxifen (Osborne et al 2003; Shou et al 2004). In addition, tamoxifen-treated patients may be exposed to a modest increased risk of vaginal bleeding, endometrial cancer, and venous thrombosis.

These limitations have prompted the search for alternative endocrine therapies with increased efficacy and fewer long-term complications and led to the development of anti-aromatase inhibitors (AIs). These compounds work by blocking the aromatase enzyme in the final step of estrogen synthesis, thus lowering circulating estrogen levels. The AIs in clinical use today include the third-generation steroidal irreversible, type I AI exemestane and the nonsteroidal reversible, type II AIs anastrozole and letrozole. The suppression of circulating estrogen is profound, approximately $95 \%$ to $98 \%$ with all third-generation AIs (Geisler et al 1998). This is the general explanation for the observed better efficacy of aromatase inhibitors compared to tamoxifen. In addition, AIs inhibit the cytochrome P450-dependent aromatase enzyme, lacking tamoxifen's partial agonist activity.

Tamoxifen is partly metabolized by CYP enzymes. Decreased CYP2D6metabolism, either because of mutations in the CYP2D6 gene and/or because of 
comedication with CYP2D6 inhibitors, may result in a decreased efficacy of the drug (Jin et al 2005). In the respective group of these individuals it is hypothesized that the use of CYP2D6 independently metabolized drugs such as AIs may be beneficial. Clinical studies to clarify this issue are ongoing.

\section{Pharmacology of exemestane}

Exemestane is an irreversible, steroidal aromatase inactivator, structurally related to the natural substrate androstenedione. It acts as a false substrate for the aromatase enzyme, and is processed to an intermediate that binds irreversibly to the active site of the enzyme causing its inactivation, an effect also known as "suicide inhibition" (Dank 2002; Dixon 2004). Exemestane significantly lowers circulating estrogen concentrations: after a single oral dose of $25 \mathrm{mg}$ the levels of estrogen, estradiol, and estrogen-sulfate decrease by $85 \%$ to $95 \%$ after 2 to 3 days and persist up to 5 days (Johannessen et al 1997). Exemestane has no detectable effect on adrenal biosynthesis of corticosteroids, aldosterone, or on other enzymes involved in the steroidogenic pathway up to a concentration at least 600 times higher than that inhibiting the aromatase enzyme (Dixon 2004).

The recommended dosage of exemestane is $25 \mathrm{mg}$ taken orally once daily after a meal which is rapidly absorbed with a maximum plasma concentration observed within 2 hours of ingestion (Evans 1992). Exemestane is distributed extensively into tissues and is $90 \%$ bound to plasma proteins, steady-state conditions are attained by 7 days (Clemett and Lamb 2000). The agent is metabolized by CYP3A4 to compounds that are inactive or show minimal inhibition of aromatase (Wong and Pritchard 2005). Excretion of exemestane is via the urine and faeces, with $<1 \%$ of the drug being excreted unchanged in urine. Although the area under the curve (AUC) of exemestane after a single 25-mg dose is elevated 3 -fold in severe renal or liver dysfunction, dose reduction is not required as the drug is well tolerated in breast cancer patients at even 8- to 24-fold higher than the recommended dose (Wong and Pritchard 2005).

\section{Exemestane in the adjuvant setting}

The efficacy of exemestane in the treatment of women with early breast cancer was evaluated in 3 large randomized trials. A third study is in progress. Exemestane was approved in the US and European Union for the adjuvant treatment of postmenopausal women with endocrine-sensitive early breast cancer who have received 2 to 3 years of tamoxifen and are switched to exemestane for the completion of a total of 5 consecutive years of adjuvant hormonal therapy.

\section{Intergroup study IES 31}

The approval was based on the data of the double-blind, randomized, adjuvant Intergroup Exemestane Study (IES) comparing 5 years of tamoxifen with the sequential use of tamoxifen followed by exemestane for a total treatment duration of 5 years. The trial included 4,742 postmenopausal patients from 37 countries and 20 co-operative groups with endocrine-sensitive early completely resected breast cancer. All of them were disease free after 2 to 3 years of tamoxifen and were randomized in the intent-to-treat (ITT) analysis to receive either tamoxifen once daily at the same dose received before randomization $(\mathrm{N}=2,372$ ) or exemestane ( $5 \mathrm{mg}$ once daily; $\mathrm{N}=2,352)$. The endocrine therapy was finished after 5 years (Figure 1 ).

The primary end point was disease-free survival (DFS), defined as the time from randomization to time of local or distant recurrence of breast cancer, contralateral invasive breast cancer, or death from any cause. Secondary end points included overall survival (OS), the incidence of contralateral breast cancer, and long- term tolerability.

The first planned interim analysis after a median follow-up of 30.6 months showed a significant improvement in DFS, relatively by $32 \%$, absolutely by $4.7 \%$ (unadjusted hazard ratio [HR] 0.68; 95\% confidence interval [CI] 0.56-0.82; $\mathrm{p}<0.001$ ) (Coombes et al 2004). DFS 3 years after randomization was $91.5 \%$ (95\% CI 90.0-92.7) in the exemestane group and $86.8 \%(95 \%$ CI 85.1-88.3) in the tamoxifen group. Furthermore, the AI proved to be significantly more efficient than tamoxifen in all subgroups, defined according to estrogen-receptor (ER) status, combined ER and progesterone-receptor status, number of positive nodes, type of previous chemotherapy, or use at any time of hormonereplacement therapy (HRT).

There was also significant superiority of exemestane in regard to distant DFS (HR 0.66; 95\% CI 0.52-0.83; $\mathrm{p}=0.0004)$ and in the risk for contralateral breast cancer (HR 0.44; $95 \%$ CI 0.20-0.98).

The updated evaluation covering a median follow-up of 55.7 months reinforced the earlier findings and demonstrated that switching to exemestane remained significantly superior to remaining on tamoxifen (Coombes et al 2007). A total of 809 events contributing to the analysis of DFS had been reported (354 exemestane, 455 tamoxifen), leading to an unadjusted HR for the ITT group for DFS of 0.76 (95\% CI 0.66-0.88; $\mathrm{p}=0.0001$ ) in favor of exemestane (Figure 2). In the ITT group, 


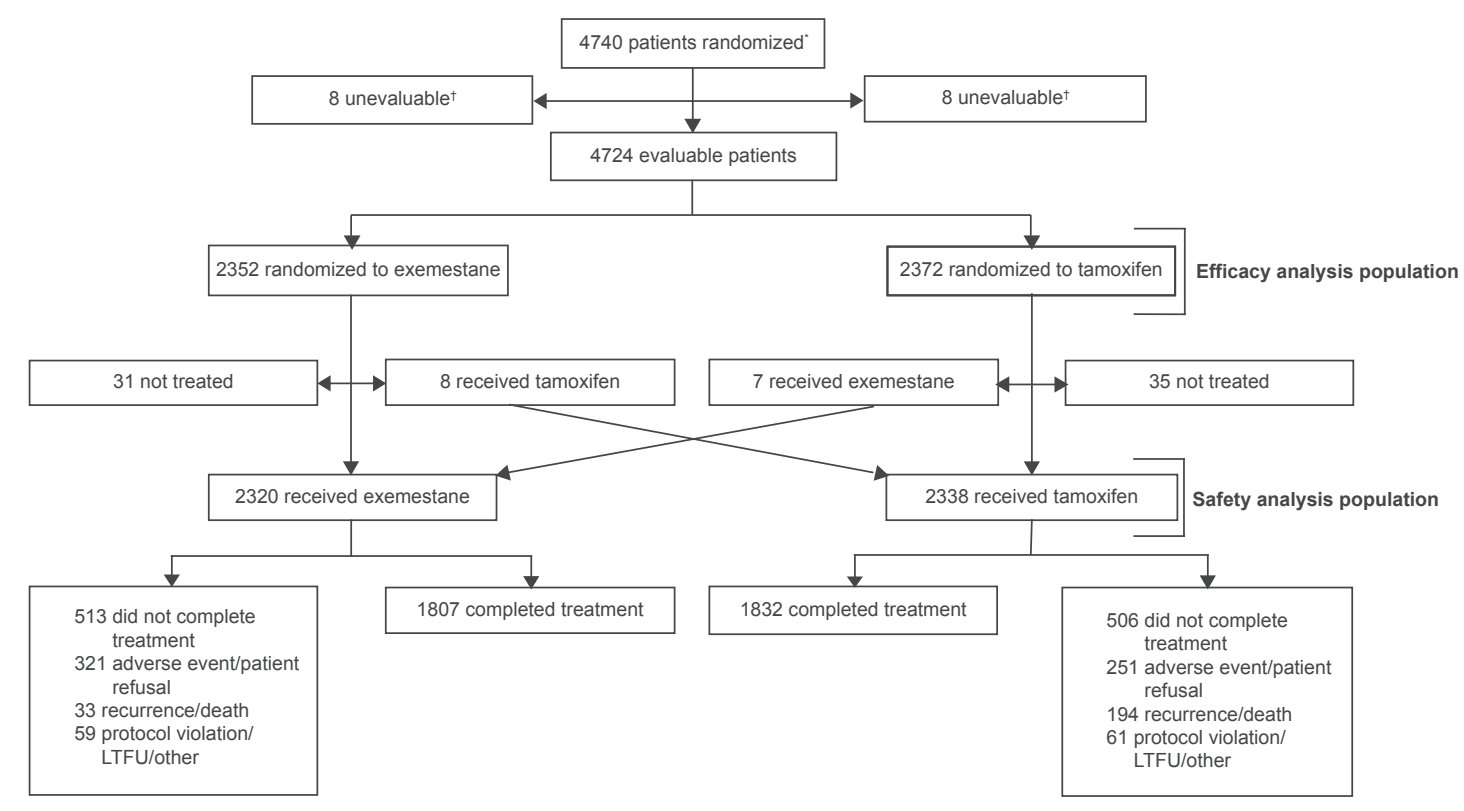

Figure 1: Trial profile

LTFU $=$ lost to follow-up. *2 patients with duplicate patient identifiers were identified, thus the total number of patients randomised is 2 fewer than proviously reported. ${ }^{+}$Monitoring for regulatory submission has resulted in an inability to confirm the validity of data at one individual center. On that basis, it was agreed that no data from patients entered at that center $(n=16)$ be used in further analyses.

Figure I IES-3I-study: trial design. Modified with permission from Coombes RC, Kilburn LS, Snowdon CF, et al 2007. Survival and safety of exemestane versus tamoxifen after 2-3 years' tamoxifen treatment (Intergroup Exemestane Study): a randomised controlled trial. Lancet, 369:559-70. Copyright @ 2007 Elsevier.

this HR translated into a $3.3 \%(95 \%$ CI 1.6-4.9) absolute improvement in DFS at 2.5 years after randomization, and a 3.4\% (0.1-6.8) improvement 5 years after randomization. Adjusting for potential confounders did not substantially affect the estimates of treatment effect, and the size of benefit for switching to exemestane was consistent across all subgroups and any tumor characteristic that was measured (Figure 3). Breast-cancer-free survival was also improved by switching to exemestane for the ITT group (HR 0.76; 95\% CI 0.65-0.89, $\mathrm{p}=0.004)$.

These data resulted in a survival benefit. In the ITT analysis 222 deaths occurred in the exemestane and 261 in the tamoxifen group, accounting for a relative risk reduction of $15 \%(p=0.08)$ (Figure 4$)$. Since the second analysis, previously unknown ER status was ascertained in 381 women. Of these, 122 were identified as having

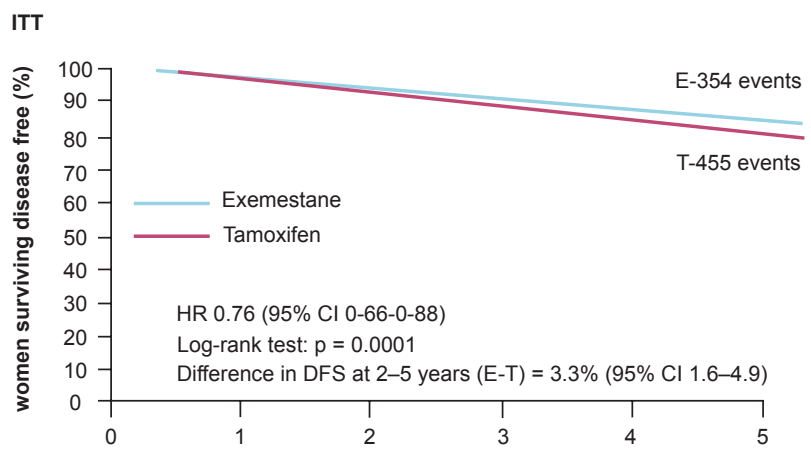

\begin{tabular}{lcr}
\multicolumn{3}{l}{ Number of events/at risk } \\
E & $0 / 2352$ & $58 / 2244$ \\
T & $0 / 2372$ & $83 / 2252$
\end{tabular}

Time since randomization (years)

$\begin{array}{llllllllll}66 / 2165 & 83 / 1954 & 70 / 1506 & 50+27^{\star} / 846 & 0 / 2296 & 55 / 2191 & 61 / 2117 & 80 / 1912 & 67 / 1474 & 49+27^{\star} / 829 \\ 104 / 2134 & 103 / 1898 & 71 / 1442 & 48+46^{*} / 812 & 0 / 2306 & 81 / 2190 & 101 / 2075 & 98 / 1848 & 67 / 1399 & 46+46^{\star} / 785\end{array}$

Figure 2 IES-3 I-study: Kaplan-Meier plots for disease-free survival (DFS; ITT-population). Modified with permission from Coombes RC, Kilburn LS, Snowdon CF, et al 2007. Survival and safety of exemestane versus tamoxifen after 2-3 years' tamoxifen treatment (Intergroup Exemestane Study): a randomised controlled trial. Lancet, 369:559-70. Copyright (C) 2007 Elsevier. 


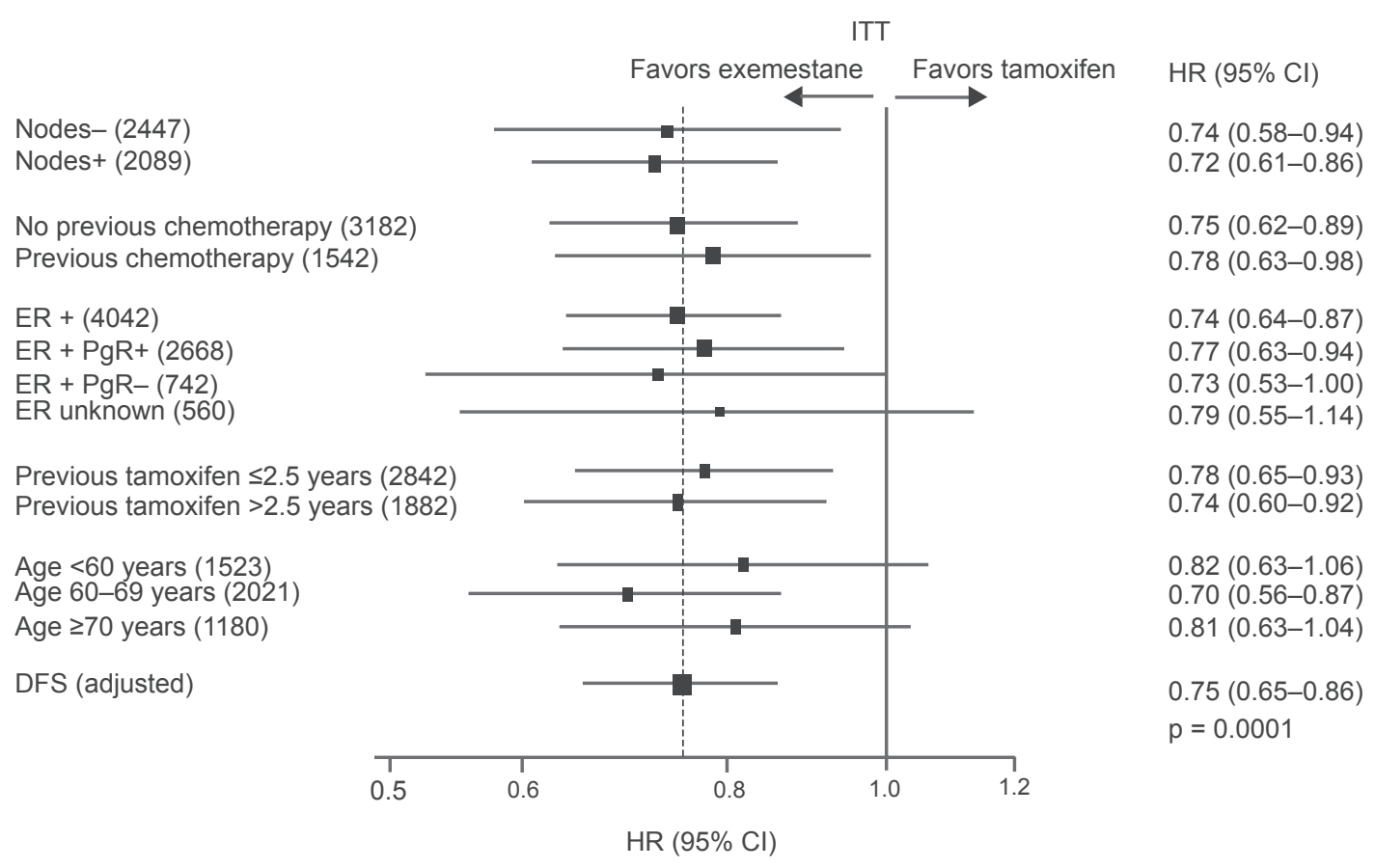

$+=$ positive,$-=$ negative. $\mathrm{ER}=$ oestrogen-receptor. $\mathrm{PgR}=$ progesterone-receptor. Forest plots display subgroup analyses depicting the $\mathrm{HR}$ as the centre of the box and $95 \% \mathrm{Cl}$ as a horizontal line, the box size being proportional to the precision of the estimate.

Figure 3 IES-3I-study: Subgroup analysis forest plot for disease-free survival (DFS; ITT-population). Modified with permission from Coombes RC, Kilburn LS, Snowdon CF, et al 2007. Survival and safety of exemestane versus tamoxifen after 2-3 years' tamoxifen treatment (Intergroup Exemestane Study): a randomised controlled trial. Lancet, 369:559-70. Copyright (C) 2007 Elsevier.

ER-negative tumors, and therefore, in addition to an ITT analysis, those with ER-negative tumors were excluded and further analysis carried out. After exclusion of ER-negative tumors and a protocol-defined adjustment for nodal status, chemotherapy use, and HRT use, the adjusted HR for death was $0.83(95 \%$ CI $0.69-0.99, \mathrm{p}=0.04)$ (Figure 4). As also shown in the second analysis, exemestane was generally well tolerated, undesirable effects were usually mild to moderate.

The clinical benefits were achieved without a detrimental effect on quality of life (QOL), as demonstrated in the IES-QOL substudy (Fallowfield et al 2006). The switch from tamoxifen to exemestane neither increased nor decreased endocrine symptoms present after 2 to 3 years of tamoxifen, and did not initiate significant new symptoms.

\section{NSABP B-33 study}

In May 2001, the National Surgical Adjuvant Breast and Bowel Project (NSABP) initiated a randomized, placebo-controlled, double-blind clinical trial to evaluate exemestane ( $25 \mathrm{mg}$ /day orally) as extended adjuvant therapy (NSABP B-33). The trial included clinical stage
$\mathrm{T}_{1-3} \mathrm{~N}_{0-1} \mathrm{M}_{0}$ endocrine-sensitive postmenopausal breast cancer patients who completed at least 5 years of tamoxifen therapy and were disease-free at the time of tamoxifen discontinuation. The primary aim of the trial was to determine whether adjuvant exemestane, for 2 years, after 5 years of tamoxifen therapy would prolong DFS compared with placebo. Secondary endpoints were OS and relapsefree survival (RFS).

When accrual to the B-33 trial was initiated, no other information existed on benefit from aromatase inhibitors in this setting. However, in October 2003, while accrual to B-33 was ongoing (1,598 patients had been accrued of 3,000 required), interim analysis results from another similarly designed trial (National Cancer Institute of Canada [NCIC] MA.17) demonstrated benefit from letrozole in patients who had completed 5 years of tamoxifen became available (Goss et al 2003). As a consequence, the accrual to B-33 was stopped, the treatment assignment unblinded, and exemestane offered to women in the placebo group. At the time of unblinding, 1,598 patients had been randomly assigned; $72 \%$ in the exemestane group continued on exemestane and $44 \%$ in the placebo group elected to receive exemestane (Figure 5). 
ITT

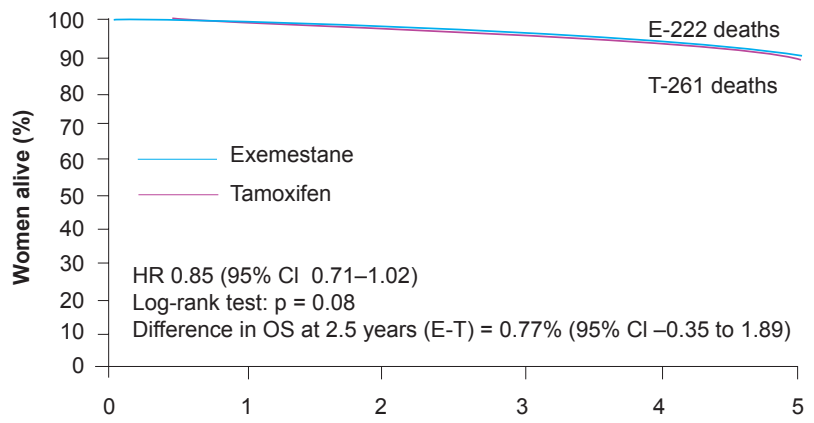

Oestrogen-receptor-positive/unknown

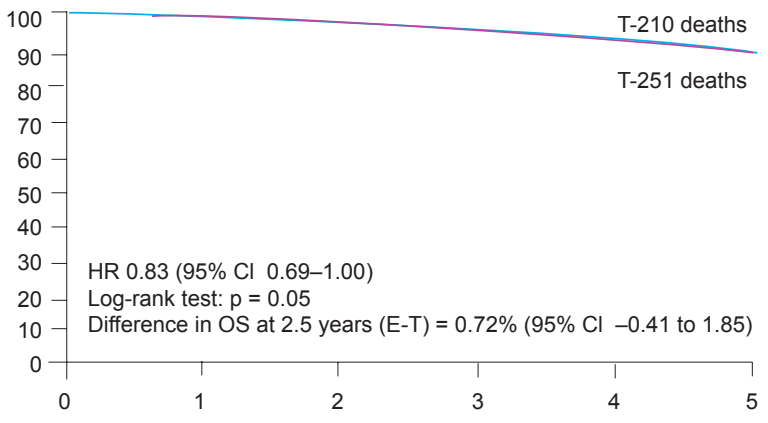

Number of events/at risk

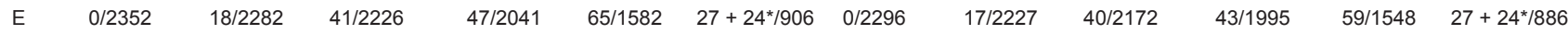

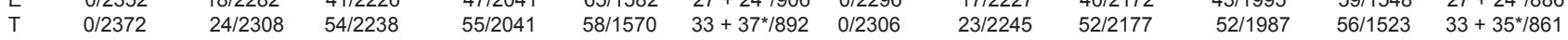

Figure 4 IES-3I-study:Kaplan-Meier plots for overall survival (OS; ITT-and oestrogen receptor positive/unknown population). Modified with permission from Coombes RC, Kilburn LS, Snowdon CF, et al 2007. Survival and safety of exemestane versus tamoxifen after 2-3 years' tamoxifen treatment (Intergroup Exemestane Study): a randomised controlled trial. Lancet, 369:559-70. Copyright (C) 2007 Elsevier.

HR $0.83(95 \% \mathrm{Cl} 0.69-0.99) \mathrm{p}=0.04$, adjustment for pre-specified prognostic factors nodal status, chemotherapy use, HRT use.

Mamounas et al (2008) published the data of the outcome analysis with 30 months of median follow-up based on the original random assignment (ITT), yielding a $32 \%$, borderline statistically significant reduction in 4-year DFS (91\% vs $89 \%$; relative risk [RR] 0.68; $p=0.07$ ) (Figure 6) and in a statistically significant $56 \%$ decrease in 4 -year RFS ( $96 \%$ vs $94 \% ; \mathrm{RR}=0.44 ; \mathrm{p}=0.004$ ). Toxicity, assessed up to time of unblinding, was acceptable for the adjuvant setting.

The reduction of DFS and RFS shown in the NSABP B 33 trial are of a magnitude similar to that seen with the nonsteroidal AIs letrozole and anastrozole in the same setting. The NCIC MA. 17 trial demonstrated a $42 \%$ reduction in recurrence with letrozole over placebo after 5 years of tamoxifen (Goss et al 2003, 2005). These findings demonstrate that exemestane may provide another option for the extended adjuvant treatment of postmenopausal women with endocrine-sensitive breast cancer who complete 5 years of adjuvant tamoxifen.

The International Breast Cancer Study Group (IBCSG) is currently evaluating the role of exemestane (IBCSG 24-02/ BIG 2-02 [SOFT]), also in combination with GnRH analogs (IBCSG 25-02/BIG 3-02 [TEXT]) as adjuvant therapy for premenopausal women with endocrine-responsive breast cancer. Both studies are ongoing and results are not available yet.

\section{Safety analysis Overall tolerability}

In general, exemestane therapy was well tolerated (Coombes et al 2004, 2007). Grade 3 or 4 adverse events were noted in $426(18.4 \%)$ patients on exemestane and $411(17.6 \%)$ on tamoxifen. At a median follow-up of 55.7 months, switching to exemestane was associated with significantly fewer thromboembolic events $(p=0.004)$, endometrial hyperplasia $(\mathrm{p}<0.0001)$, uterine polyps $(\mathrm{p}<0.0001)$, and serious gynecological events $(p=0.0002)$ than continuing on tamoxifen.

Musculoskeletal pain, carpal tunnel syndrome, joint stiffness, paraesthesia, and arthralgia were reported more frequently in patients who switched to exemestane than in those who remained on tamoxifen. These effects emerged during the on-treatment period.

Myocardial infarctions were rare and occurred in $31(1.3 \%)$ exemestane-treated patients compared with $19(0.8 \%)$ tamoxifen-treated patients $(\mathrm{p}=0.08)$. Any effect of treatment on risk of myocardial infarction seemed largely restricted to patients with a history of hypertension.

\section{Menopausal symptoms}

The phase III, randomized Tamoxifen Exemestane Adjuvant Multicenter (TEAM) trial is a prospective open-label randomized multicenter study designed specifically to evaluate the efficacy of upfront adjuvant exemestane therapy for endocrine-sensitive postmenopausal early breast cancer in comparison with a crossover design using exemestane for 2 to 3 years after 2 to 3 years of tamoxifen. Patients received either tamoxifen $20 \mathrm{mg}$ once daily orally for 2.5 or 3 years followed by exemestane $25 \mathrm{mg}$ once daily for 2 or 2.5 years, or exemestane $25 \mathrm{mg}$ once daily for 5 years, as a double-blind capsule for year 1, and open label for 


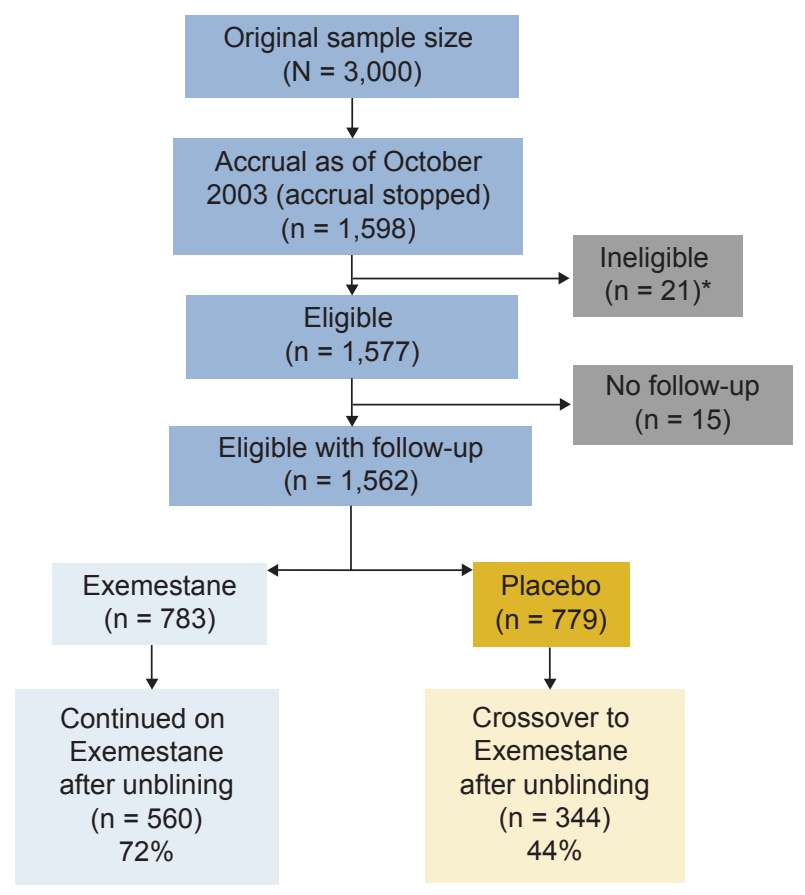

$\left({ }^{*}\right)$ Reasons for ineligibility: abnormal pre-entry studies $(n=2)$; required pre-entry studies not performed $(n=4)$; or not performed as specified $(n=5)$; premenopausal status $(n=1)$; prior breast cancer other than lobular carcinoma in situ $(n=2)$; prior recurrence $(n=1)$; unknown/negative estrogen-/progesterone-receptor status $(n=3)$; tamoxifen therapy outside the prespecified range $(n=1)$; original tumor not invasive carcinoma $(n=1)$; unknown margin status $(n=1)$.

Figure 5 NSABP B-33-study:CONSORT diagram. Modified with permission from Mamounas EP, Jeong JH, Wickerham DL, et al. 2008. Benefit from exemestane as extended adjuvant therapy after 5 years of adjuvant tamoxifen:intention-to-treat analysis of the National Surgical Adjuvant Breast And Bowel Project B-33 trial.J Clin Oncol, 26:1965-7I. Copyright (C) 2008 American Society of Clinical Oncology.

4 years. Adjuvant hormonal treatment was initiated within 10 weeks after surgery or completion of chemotherapy, whichever occurred last. The primary endpoint was defined as DFS, secondary endpoints included OS, incidence of new primary breast cancers, and safety parameters. In addition, 5 substudies were used to separately analyze endometrial changes, lipid profiles, QOL, tolerability of therapy, and bone changes. A meta-analysis of overall drug efficacy will use data from the combined substudies, but has not been published yet. However, results of the menopausal substudy as well as the bone data are available.

Jones et al (2007) assessed the prevalence of 10 common menopausal symptoms associated with either adjuvant tamoxifen or exemestane by self-report questionnaire administered to 1,614 consecutive patients at baseline and every 3 months during the first year of the trial. Symptoms were categorized as none, mild, moderate, or severe. A hot flash score was calculated at each time point. Symptoms were analyzed by repeated-measures analysis of variance.
Each time period was tested repeatedly against the baseline; an overall p-value was assigned for each reported symptom. The strengths of this study were the large number of women evaluated $(\mathrm{N}=1,614)$, excellent compliance (7,286 completed questionnaires; $81 \%$ completion at 12 months), and the double-blinded design.

The TEAM study showed at 12 months that patients receiving tamoxifen, versus those receiving exemestane, had a significantly higher mean hot flush score $(\mathrm{p}=0.0253)$ and more vaginal discharge $(\mathrm{p}<0.0001)$, whereas women under exemestane had more vaginal dryness $(p=0.0004)$ and decreased libido $(\mathrm{p}=0.0343)$.

Thomas et al (2008) reported in an open-label, prospective, crossover study with 184 post-menopausal women experiencing hot flushes on adjuvant tamoxifen that 6 weeks after switching to letrozole or exemestane, the primary end point, hot flush score, improved by $47.3 \%(\mathrm{p}<0.001)$ compared to those reported on tamoxifen. The mean mood rating scale (MRS) score improved by $9.7 \%(\mathrm{p}=0.01)$. At 6 weeks, significantly more women chose to remain on an AI (72\% vs $22 \%$ preferring tamoxifen; $\mathrm{p}<0.001)$. At 3 months, 107 women (58\%) preferred to remain on an AI, $55(30 \%)$ on tamoxifen, and $22(12 \%)$ withdrew. These data suggest that in women suffering significant adverse effects on tamoxifen, switching to an AI like exemestane improves hot flushes, mood, and quality of life.

\section{Bone metabolism}

The effects of a profound long-term estrogen deprivation achievable with the third-generation aromatase inhibitors in respect to the bone health has been a major concern.

Unlike nonsteroidal AIs, exemestane may exert beneficial effects on bone through its primary metabolite 17-hydroexemestane which expresses androgen agonistic activity. In fact, a preclinical study reported that the administration of exemestane to ovariectomized rats reduced both bone formation and resorption markers and attributed this pattern to the androgenicity of exemestane and its principal metabolite (Goss et al 2004). Lønning et al (2005) reported a significant increased serum level and urinary excretion of bone resorption, but also bone formation markers in postmenopausal women with early breast cancer under exemestane $25 \mathrm{mg}$ daily for 2 years in a double-blind setting which could be due to this androgenic effect.

In contrast, a subgroup of the IES 31-study focusing on the effect on bone health showed that within 6 months of switching to exemestane, bone mineral density (BMD) 
was lowered by $0.051 \mathrm{~g} / \mathrm{cm}^{3}(2.7 \% ; 95 \%$ CI $2.0-3.4$; $\mathrm{p}<0.0001)$ at the lumbar spine and $0.025 \mathrm{~g} / \mathrm{cm}^{3}(1.4 \%$; $0.8-1.9 ; \mathrm{p}<0.0001)$ at the hip compared with baseline (Coleman et al 2007). However, BMD decreases were only $1.0 \%(0.4-1.7 ; \mathrm{p}=0.002)$ and $0.8 \%(0.3-1.4 ; \mathrm{p}=0.003)$ in year 2 at the lumbar spine and hip, respectively (Figure 7). Further decreases were smaller and approached rates observed in a healthy age-matched population. No patient with BMD in the normal range at trial entry developed osteoporosis. Bone resorption and formation markers increased at all time points in women receiving exemestane $(p<0.001)$. With a median follow-up in all IES participants $(n=4,274)$ of 58 months, $162(7 \%)$ and $115(5 \%)$ patients in the exemestane and tamoxifen groups, respectively, had fractures (odds ratio 1.45 [1.13-1.87]; $\mathrm{p}=0.003)$.

These findings are in accordance with the data of another study in postmenopausal patients switched to exemestane after 2 to 3 years of adjuvant treatment with tamoxifen, showing a marked increase in bone turnover markers with a consequent reduction in BMD (Gonelli et al 2007). This pattern of BMD is more evident at skeletal sites where trabecular bone prevails and is most likely due to rapid cessation of bone turnover suppression by tamoxifen.

These findings corroborate the results of the bone TEAM-substudy, evaluating 161 women receiving tamoxifen $(\mathrm{N}=78)$ or exemestane $(\mathrm{N}=83)$, showing a loss in absolute BMD under exemestane at 12 months (Hadji et al 2008). The difference was significant at the spine $(\mathrm{p}=0.0008$ and $\operatorname{hip}(\mathrm{p}=0.04)$. However, during the second year of treatment, the rate of BMD loss slowed dramatically. All measured markers of bone formation and resorption show an increase with exemestane.

\section{Lipid profile}

Given their mechanism of action, one might expect that AIs may have certain adverse effects on multiple estrogen-dependent metabolic functions such as lipid metabolism when compared with tamoxifen.

The benefits of receiving an AI are likely to outweigh the disadvantages of any changes to lipid profiles. However, when these compounds are used in early disease or in the prevention setting, an increase of $10 \%$ to $15 \%$ in circulating cholesterol and triglycerides may have a significant impact on the risk of cardiovascular disease, and monitoring of blood lipid levels and instigation of lipid lowering treatment if required should be undertaken (Bundred 2005).

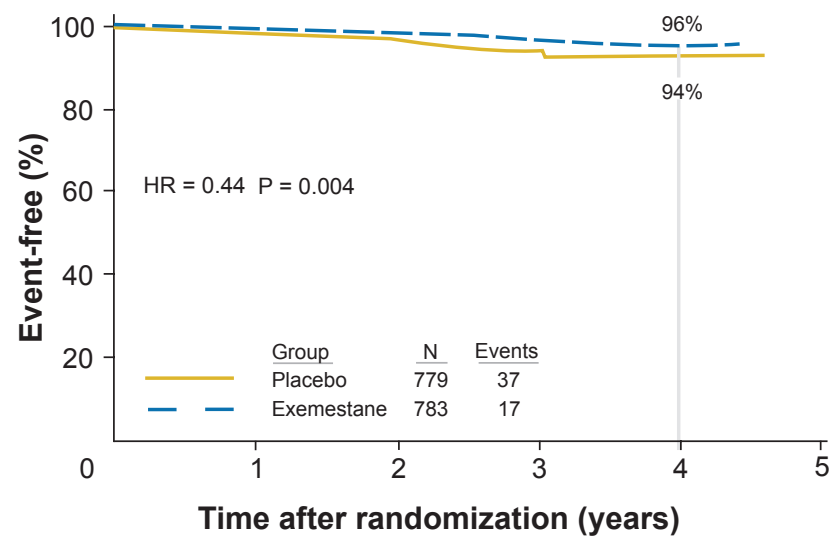

$\begin{array}{lllll}\text { No. at risk } & & & & \\ \text { Placebo } & 743 & 562 & 162 & 16 \\ \text { Exemestane } & 752 & 599 & 196 & 15\end{array}$

Figure 6 NSABP B-33-study: Kaplan Meier-estimates of relapse-free survival (RFS) (ITT for eligible patients with follow-up). Modified with permission from Mamounas EP, Jeong JH,Wickerham DL, et al. 2008. Benefit from exemestane as extended adjuvant therapy after 5 years of adjuvant tamoxifen:intention-to-treat analysis of the National Surgical Adjuvant Breast And Bowel Project B-33 trial. J Clin Oncol, 26:1965-7I. Copyright (C) 2008 American Society of Clinical Oncology.

A subprotocol to the ATENA (Adjuvant postTamoxifen Exemestane versus Nothing Applied) trial compared the effect of exemestane on the lipid profile in the adjuvant setting to that of observation alone following deprivation of 5 to 7 years primary treatment with tamoxifen (Markopoulos et al 2005). In this open-label, randomized, parallel group study, 340 post-menopausal women with operable breast cancer who had been treated with tamoxifen for 5 to 7 years were randomized to either 5 additional years of exemestane ( $25 \mathrm{mg} /$ day; $\mathrm{N}=172$ ) or observation alone $(\mathrm{N}=168)$.

Total serum triglycerides (TRG) levels were significantly reduced compared with baseline for the exemestane and the observational arm. Both total cholesterol and low-density lipoprotein (LDL) concentrations significantly increased above that of baseline values by 6 months, maintained through to 12 months, with no significant difference between the two treatment arms. There was no significant alteration observed for high-density lipoprotein (HDL) over time or between the two arms. In conclusion, switching patients to adjuvant exemestane treatment does not induce major effects on serum lipids.

\section{Endometrial effects}

A prospective open label randomized multicenter substudy was performed in the German TEAM Trial Group to test the hypothesis that the irreversible inactivation of aromatase by exemestane (EXE; $\mathrm{N}=78$ ) would result in less endometrial 
proliferation than tamoxifen (TAM; $\mathrm{N}=65$ ) (Kieback et al 2006).

After a median follow-up of 727 (EXE) and 526 days (TAM), endometrial hyperplasia was observed in 11 patients in the exemestane-arm versus 45 patients under tamoxifen $(p<0.0001)$. Time to endometrial hyperplasia was significantly longer in the EXE group $(\mathrm{p}<0.0001)$; HR was 0.160 indicating an $84 \%$ risk reduction of hyperplasia in the EXE group. Only one patient underwent histological sampling in the EXE group (no hyperplasia) versus 18 in the TAM subset.

\section{Cost effectiveness}

Thompson et al (2007) performed an analysis of the costeffectiveness of switching to exemestane versus staying on tamoxifen based on the IES data. Because the trial data span a limited period of time and do not include information on resource utilization, the authors used modeling techniques and data from sources external to the IES to project long-term survival and economic costs.

A Markov model was developed to predict patients' transitions across various health states based on treatment strategy (continuing tamoxifen vs switching to exemestane), breast cancer status (no recurrence, local or distant recurrence, contralateral breast cancer), and other related health events (osteoporosis, endometrial cancer, death). Rates of disease-related events (recurrence and contralateral breast cancer) were estimated using data from the IES. Survival and lifetime medical-care costs by type of disease-related event were estimated using SEER-Medicare data. The model was used to estimate direct costs (in 2004 US\$7,724 per patient), life expectancy, quality-adjusted life-years (QALYs), and incremental cost-effectiveness.

Switching to exemestane versus continuing tamoxifen therapy was associated with an increased disease-free survival, QALYs (12.21 vs 11.89), and net discounted lifetime costs of cancer care $(\$ 12,124$ vs $\$ 7,724$ per patient). The incremental cost-effectiveness ratio of exemestane was $\$ 20,100$ per QALY gained $(95 \%$ CI $\$ 12,100, \$ 59,000)$. According to the authors' conclusion, the switch-to-exemestane strategy versus completing a 5-year course of tamoxifen is a costeffective use of health-care resources, despite the fact that the per-day cost of exemestane therapy is nearly 3 times higher than that of tamoxifen.

\section{Conclusion for clinical implications}

The data of the IES 31-trial demonstrated that switching to exemestane after 2 to 3 years of tamoxifen significantly improves disease-free survival and reduces the chance of
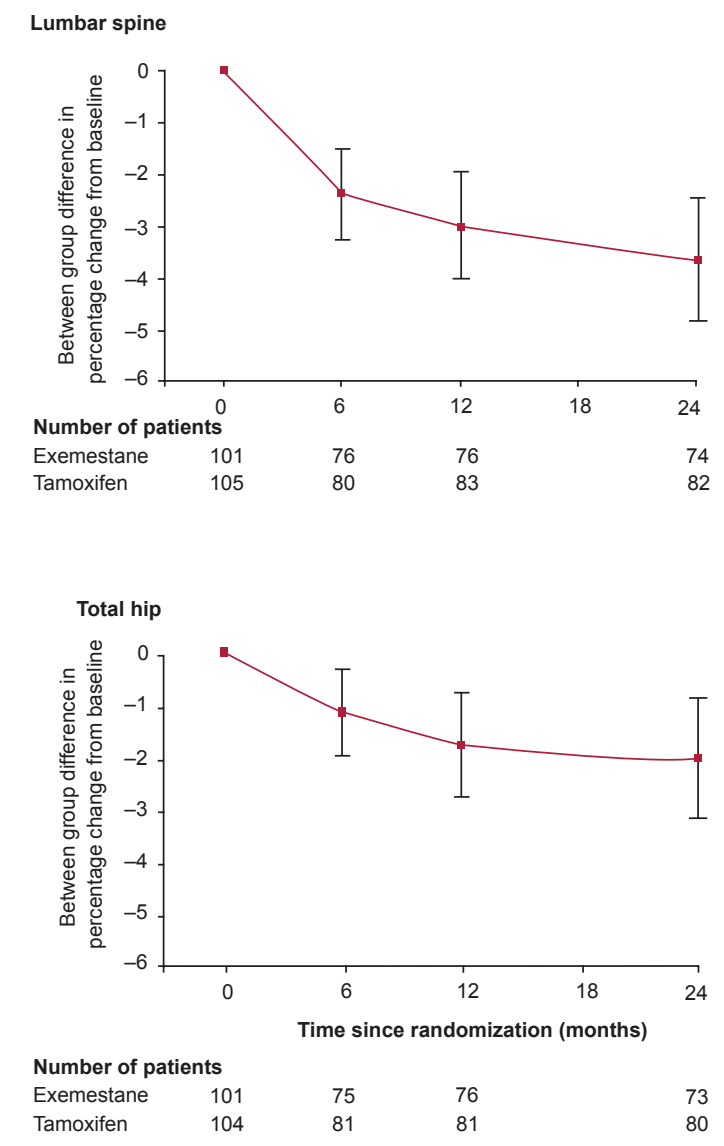

Figure 7 Difference in mean percentage change from baseline BMD (with $95 \% \mathrm{Cls}$ ) between the exemestane and tamoxifen treatment groups at lumbar spine and total hip. Modified with permission from Coleman RE, Banks LM, Girgis SI, et al. 2007. Skeletal effects of exemestane on bone-mineral density, bone biomarkers, and fracture incidence in postmenopausal women with early breast cancer participating in the Intergroup Exemestane Study (IES): a randomised controlled study. Lancet Oncol, 8:1 19-27. Copyright (C) 2007 Elsevier.

new disease in the contralateral breast or distant recurrence, independent of any tumor characteristics (Coombes et al 2007). As shown in the updated results, the early improvements in DFS noted in patients who switched to exemestane persist after treatment, and translate into a modest improvement in overall survival. In addition, the findings of the IES also demonstrate that the benefit of switching from tamoxifen to an AI in patients with endocrine-responsive breast cancer persists for some years after discontinuation of the AI.

So far, no data are available from trials that directly compare use of AI monotherapy with a sequential or switch strategy. Therefore, direct evidence from the sequential therapy arms of BIG 1-98 and from the TEAM trial 18 is awaited with keen interest to conclude on the value of the sequential strategy.

Exemestane appears to be different from other aromatase inhibitors with its less detrimental effect on bone after 
12 months of treatment. However, the available data do not enable confirmation that exemestane may have any substantial advantageous effects on bone with respect to the nonsteroidal aromatase inhibitors (Gonelli et al 2007). The data suggest that postmenopausal women switched from tamoxifen to exemestane should be monitored for bone loss in accordance with the American Society of Clinical Oncology recommendations (Hillner et al 2003), especially if other risk factors for osteoporosis are present.

The national (ie, AGO Breast Commission) and international guidelines recommend 3 strategies:

1. upfront treatment with letrozole or anastrozole

2. switching to exemestane or anastrozole after 2 to 3 years of tamoxifen

3. extended adjuvant treatment with letrozole after completion of 5 years of tamoxifen

To date, the data are in favor of both upfront AIs or switch to an $\mathrm{AI}$ in patients already on tamoxifen for 2 to 3 years not having relapsed while on tamoxifen.

\section{Acknowledgment}

We thank Dr Katrina Recker, Hamburg, for the editorial assistance.

\section{Disclosures}

The authors have no conflicts of interest to disclose.

\section{References}

Bundred NJ. 2005. The effects of aromatase inhibitors on lipids and thrombosis. Br J Cancer, 93(Suppl 1):S23-7.

Clemett N, Lamb NB. 2000. Exemestane:a review of its use in post-menopausal women with breast cancer. Drugs, 59:1279-96.

Coleman RE, Banks LM, Girgis SI, et al. 2007. Skeletal effects of exemestane on bone-mineral density, bone biomarkers, and fracture incidence in postmenopausal women with early breast cancer participating in the Intergroup Exemestane Study (IES): a randomised controlled study. Lancet Oncol, 8:119-27.

Coombes RC, Hall E, Gibson LJ, et al. 2004. A randomized trial of exemestane after two to three years of tamoxifen therapy in postmenopausal women with primary breast cancer. $N$ Engl $J$ Med, 350:1081-92.

Coombes RC, Kilburn LS, Snowdon CF, et al. 2007. Survival and safety of exemestane versus tamoxifen after 2-3 years' tamoxifen treatment (Intergroup Exemestane Study): a randomised controlled trial. Lancet, 369:559-70.

Dank M. 2002. The role of aromasin in the hormonal therapy of breast cancer. Pathol Oncol Res, 2:87-92.

Dixon JM. 2004. Exemestane and aromatase inhibitors in the management of advanced breast cancer. Expert Opin Pharmacother, 5:307-16.

Early Breast Cancer Trialists' Collaborative Group (EBCTCG). 2005. Effects of chemotherapy and hormonal therapy for early breast cancer on recurrence and 15-year survival:an overview of the randomised trials. Lancet, 365:1687-717.

Early Breast Cancer Trialists' Collaborative Group. 1998. Tamoxifen for early breast cancer:and overview of the randomised trials. Lancet, 351:1451-67.
Evans TR, Disalle E, Ornati G, et al. 1992. Phase I and endocrine study of exemestane (FCE 24304), a new aromatase inhibitor, in postmenopausal women. Cancer Res, 52:5933-39.

Fallowfield LJ, Bliss JM, Porter LS, et al. 2006. Quality of life in the Intergroup Exemestane Study: A randomized trial of exemestane versus continued tamoxifen after 2 to 3 years of tamoxifen in postmenopausal women with primary breast cancer. J Clin Oncol, 24:910-7.

Geisler J, King N, Anker G, et al. 1998. In vivo inhibition of aromatization by exemestane, a novel irreversible aromatase inhibitor, in postmenopausal breast cancer patients. Clin Cancer Res, 4:2089-93.

Gonnelli S, Cadirni A, Caffarelli C, et al. 2007. Changes in bone turnover and in bone mass in women with breast cancer switched from tamoxifen to exemestane. Bone, 40:205-10.

Goss PE, Ingle JN, Martino S, et al. 2003. A randomized trial of letrozole in postmenopausal women after five years of tamoxifen therapy for early-stage breast cancer. $N$ Engl J Med, 349:1793-802.

Goss PE, Ingle JN, Martino S, et al. 2005. Random-ized trial of letrozole following tamoxifen as extended adjuvant therapy in receptor-positive breast cancer:Updated findings from NCIC CTG MA. 17. J Natl Cancer Inst, 97:1262-71.

Goss PE, Qi S, Josse RG, Pritzker KP, et al. 2004. The steroidal aromatase inhibitor exemestane prevents bone loss in ovariectomized rats. Bone, 34:384-92.

Hadji P, Ziller M, Kieback DG et al. 2008. Bone effects of exemestane vs tamoxifen within the TEAM trial:results of a prospective randomized bone sub-study. J Clin oncol, 26(May 20 suppl):Abstr 572.

Hillner BE, Ingle JN, Chlebowski RT, et al. 2003. American Society of Clinical Oncology 2003 update on the role of bisphosphonates and bone health issues in women with breast cancer. J Clin Oncol, 21:4042-57.

Iddon J, Bundred NJ. 2008. To switch or not to switch:should the updated Intergroup Exemestane Study alter our decision? Expert Rev Anticancer Ther, 8:9-13.

Jin Y, Desta Z, Stearns V, et al. 2005. CYP2D6 genotype, antidepressant use, and tamoxifen metabolism during adjuvant breast cancer treatment. J Natl Cancer Inst, 97:30-9.

Johannessen DC, Engan T, diSalle E, et al. 1997. Endocrine and clinical effects of exemestane (PNU 155971), a novel steroidal aromatase inhibitor, in postmenopausal breast cancer patients:a Phase I study. Clin Cancer Res, 3:1101-8.

Jones SE, Cantrell J, Vukelja S, et al. 2007. Comparison of menopausal symptoms during the first year of adjuvant therapy with either exemestane or tamoxifen in early breast cancer:report of a Tamoxifen Exemestane Adjuvant Multicenter trial substudy. J Clin Oncol, 25:4765-71.

Kieback DG, Harbeck N, Bauer W. 2007. Endometrial effects of exemestane compared to tamoxifen within the TEAM trial:Results of a prospective randomized study. J Clin Oncol, ASCO Annual Meeting Proceedings 25(18S, June 20 Suppl):572.

Lønning PE, Geisler J, Krag LE, et al. 2005. Effects of exemestane administered for 2 years versus placebo on bone mineral density, bone biomarkers, and plasma lipids in patients with surgically resected early breast cancer. J Clin Oncol, 23:5126-37.

Mamounas EP, Jeong JH, Wickerham DL, et al. 2008. Benefit from exemestane as extended adjuvant therapy after 5 years of adjuvant tamoxifen:intention-to-treat analysis of the National Surgical Adjuvant Breast And Bowel Project B-33 trial. J Clin Oncol, 26:1965-71.

Markopoulos C, Chrissochou M, Michailidou A, et al. 2005. Effect of exemestane on the lipidemic profile of post-menopausal operable breast cancer patients following 5-7 years of adjuvant tamoxifen:preliminary results of the ATENA substudy. Anticancer Drugs, 16:879-83.

Osborne CK, Bardou V, Hopp TA, et al. 2003. Role of the estrogen receptor coactivator AIB1 (SRC-3) and HER-2/neu in tamoxifen resistance in breast cancer. J Natl Cancer Inst, 95:353-61.

Saphner T, Tormey DC, Gray R, et al. 1996. Annual hazard rates of recurrence for breast cancer after primary therapy. J Clin Oncol, $14: 2738-46$ 
Shou J, Massarweh S, Osborne CK, et al. 2004. Mechanisms of tamoxifen resistance:Increased estrogen-receptor-HER2/neu cross-talk in ER/HER2-positive breast cancer. J Natl Cancer Inst, 96:926-35.

Thomas R, Williams M, Marshall C, Walker L, 2008. Switching to letrozole or exemestane improves hot flushes, mood and quality of life in tamoxifen intolerant women. Br J Cancer, 98:1494-9.
Thompson D, Taylor D, Montoya E et al. 2007. Cost-Effectiveness of Switching to Exemestane after 2 to 3 Years of Therapy with Tamoxifen inPostmenopausal Women with Early-Stage Breast Cancer. Value Health, 5:367-76.

Wong NS, Pritchard KI, 2005. A review of exemestane in the management of breast cancer. Expert Opin Pharmacother, 6:2353-63. 